\title{
Probabilistic fatigue analysis of a post-weld treated tubular bridge
}

\author{
S. Walbridge \& A. Nussbaumer \\ Swiss Federal Institute of Technology Lausanne, ICOM - Steel Structures Laboratory, Lausanne, Switzerland
}

\begin{abstract}
In this paper, several probabilistic models for evaluating the fatigue performance of post-weld treated tubular bridge structures are briefly described. The application of the models is then demonstrated on a full-scale example bridge. Under realistic traffic loading conditions, it is shown that a significant increase in fatigue performance can be obtained with post-weld treatment. In comparing several treatment strategies, a partial treatment strategy is presented, which results in the same fatigue performance improvement as full treatment with a fraction of the treatment effort. One potential concern with the use of post-weld treatment methods such as needle peening is that they may simply shift the critical crack site to an untreatable location such as the weld root. To address this concern, a deterministic verification of this fatigue detail is presented. Shortcomings of this verification are then discussed, and future research needs highlighted.
\end{abstract}

\section{INTRODUCTION}

Bridges consisting of steel tubes welded together to form truss girders represent an industry trend that has received much recent attention from practicing engineers, code writing authorities, and researchers. The merits of these structures are cited in a number of references. One of their often cited weaknesses, and thus an area meriting further study, is the relatively poor fatigue performance of their joints.

Two ways of improving this fatigue performance have been considered in recent studies: 1) replacing the directly welded joints with cast steel nodes, and 2) improving the performance of the fatigue-critical welds by post-weld treatment (PWT).

The use of residual stress-based PWT methods such as needle or hammer peening to improve the fatigue performance of tubular bridge joints has recently been investigated in a laboratory test-based study (Schumacher 2003) and a subsequent probabilistic fracture mechanics-based study (Walbridge et al. 2003, Walbridge 2005). This paper presents a number of key findings from this second study.

Specifically, probabilistic models developed in this second study are used to analyze a full-scale tubular bridge structure under realistic loading conditions. In Section 2 of this paper the probabilistic models used for this analysis are briefly described. Following this, an example bridge structure is described and the results of a deterministic, code-based fatigue verification of this structure are presented. A probabilistic analysis of the example structure is then performed. With the results of this analysis, the deterministic verification procedure is validated and the benefit of post-weld treatment by needle peening is evaluated. Finally, in order to assess the implications of fatigue cracking at the weld root, a deterministic verification of this fatigue detail is presented. Shortcomings of this verification are then discussed, and future research needs highlighted.

\section{PROBABILISTIC MODEL OVERVIEW}

The probabilistic models employed herein allow the probabilities of fatigue failure of single potential crack sites (i.e. hot-spots) in tubular joints and entire tubular truss structures with multiple potential crack sites to be determined at various points during the life of the structure. The details of these models are presented in Appendix I. The basis for the single site model is a linear elastic fracture mechanics-based approach. The model has been developed for applications involving non-over lapping single circular hollow section (CHS) K-joints (see Fig. 1) such as those common to planar Warren trusses.

The basis for the multiple site model employed herein is a systems reliability-based approach, wherein the entire tubular joint or truss is considered as a simple series system (see also Appendix I).

For the probabilistic analysis of each potential crack site, the required input includes the following parameters: the initial defect depth, $a_{0}$, the initial defect shape, $(a / c)_{0}$, the critical crack depth, $a_{c}$, the 
crack propagation parameters: $C, m$, and $\Delta K_{t h}$, the various parameters describing the joint geometry (see Fig. 1), the weld angle and footprint length, $\theta_{w}$ and $L_{w}$, the hot-spot stress, $\sigma_{h s}$, due to the dead load, and a range-mean histogram of the hot-spot stresses due to the passage of vehicles over the bridge.

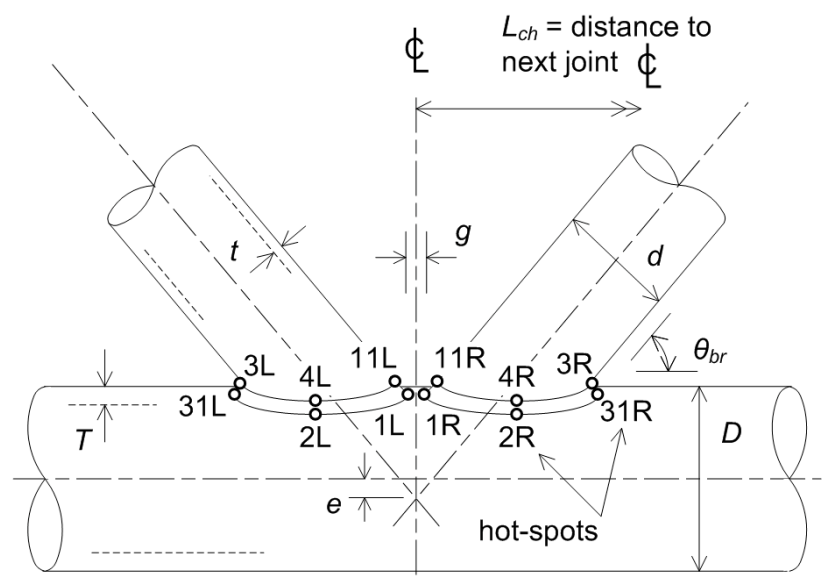

Figure 1. Non-overlapping single K-joint.

Among these parameters, $a_{c}, m$, and the joint geometry parameters are treated deterministically, while the parameters $a_{0},(a / c)_{0}, C$, and $\Delta K_{t h}$ are described by statistical variables. Several additional statistical variables are introduced in the form of factors to consider uncertainties associated with: the dead load and traffic induced hot-spot stresses, the weld angle and footprint length, and a number of other parameters contained in the crack propagation model including the magnification and correction factors, $M k$ and $Y$, stress concentration factor, $S C F$, and degree of bending, $D O B$ (see Appendix I).

Along with these deterministic parameters and statistical variables, additional parameters are introduced to describe the residual stresses along the anticipated crack path due to: 1) the welding process, and 2) the subsequent post-weld treatment application. These parameters are also discussed in greater detail in Appendix I. Herein, it is assumed that treatments such as needle peening have the primary effect of introducing compressive residual stresses near the treated surface, which tend to reduce crack propagation rates at smaller crack depths.

\section{DESCRIPTION OF BRIDGE STRUCTURE}

The characteristics of the example bridge structure studied herein were established based on a survey of existing tubular truss bridges. These characteristics can be summarized as follows (see Fig. 2):

- highway bridge with 3 x $40 \mathrm{~m}$ spans supporting two opposing lanes of traffic,

- cross section consisting of an $11 \mathrm{~m}$ wide concrete slab sitting on two planar tubular trusses (full composite action assumed), and
- Warren truss configuration assumed with single CHS K-joints $\left(\theta_{b r}=55^{\circ}, 12\right.$ truss bays per span).

The bridge was designed based on a deterministic code-based fatigue verification carried out in accordance with the requirements of the Swiss SIA Codes (2003), including the following assumptions:

- Planned service life $=70$ years.

- 40 tonne legal truck weight limit.

- Principal road traffic $\rightarrow 5 \cdot 10^{5}$ trucks/dir./year.

The design truck used in the SIA (2003) code-based fatigue verification consists of two $270 \mathrm{kN}$ axle loads spaced $1.2 \mathrm{~m}$ apart. The verification consists of evaluating the following relationship:

$\Delta \sigma_{E 2} \leq \frac{\Delta \sigma_{c, t}}{\gamma_{M f}}$

where $\Delta \sigma_{E 2}$ is the equivalent design hot-spot stress range at $2 \cdot 10^{6}$ cycles, calculated as follows:

$\Delta \sigma_{E 2}=\lambda_{1} \cdot \Delta \sigma\left(Q_{\text {fat }}\right)$

where $\Delta \sigma\left(Q_{f a t}\right)$ is the hot-spot stress range, $\Delta \sigma_{h s}$, at the location of interest due to a single passage of the design truck and $\lambda_{1}$ is a damage equivalence factor $\left(\lambda_{1}=1.42\right.$ for a $40 \mathrm{~m}$ bridge span on a principal road). In Equation 1, $\Delta \sigma_{c, t}$ is the fatigue strength corresponding with $2 \cdot 10^{6}$ applied stress cycles. According to Schumacher (2003), $\Delta \sigma_{c, 20}=86 \mathrm{MPa}$ should be used for the hot-spot stress-based design of tubular bridge joints, with a reference wall thickness of $20 \mathrm{~mm}$, and the following size effect correction:

$\frac{\Delta \sigma_{c, t}}{\Delta \sigma_{c, 20}}=\left(\frac{20}{T \text { or } t}\right)^{0.25}$

In Equation 1, $\gamma_{M f}$ takes on a value between 1.0 and 1.35 depending on the ease with which fatigue damage may be detected / repaired and the consequence of fatigue failure. For each joint along the bottom chord of the interior span, this verification was carried out for each of the hot-spots in Figure 1.

Under a given set of loads, the hot-spot stress, $\sigma_{h s}$, at a given potential crack site or hot-spot can be determined using the following expression:

$$
\begin{aligned}
\sigma_{h s}= & \sigma_{a x \_b r} \cdot S C F_{a x \_b r}+\sigma_{a x \_c h} \cdot S C F_{a x \_c h} \\
& +\sigma_{i p b 1 \_b r} \cdot S C F_{i p b 1 \_b r}+\sigma_{i p b 2 \_b r} \cdot S C F_{i p b 2 \_b r} \\
& +\sigma_{i p b \_c h} \cdot S C F_{i p b_{-} c h}
\end{aligned}
$$

where $\sigma_{a x b r}$ is the nominal member stress due to balanced axial brace load case; and $S C F_{a x} b r$ is the corresponding stress concentration factor, etc. The five load cases implicated in Equation 4 are shown in Figure 3. The associated nominal member stresses can be determined by structural analysis.

In the current study, the $S C F$ s in Equation 4 were determined using tables for CHS K-joints from (Schumacher 2003). These tables require as input the hot-spot location and the following parameters: $\beta(=d / D), \gamma(=0.5 \cdot D / T), \tau(=t / T)$, and $\theta_{b r}$. 


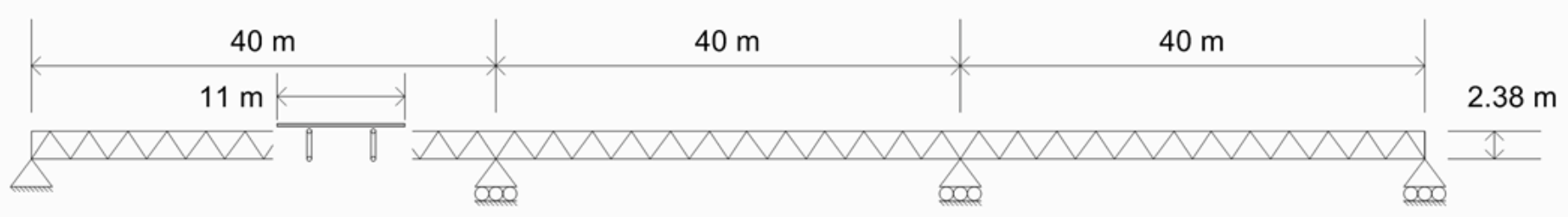

Interior span:

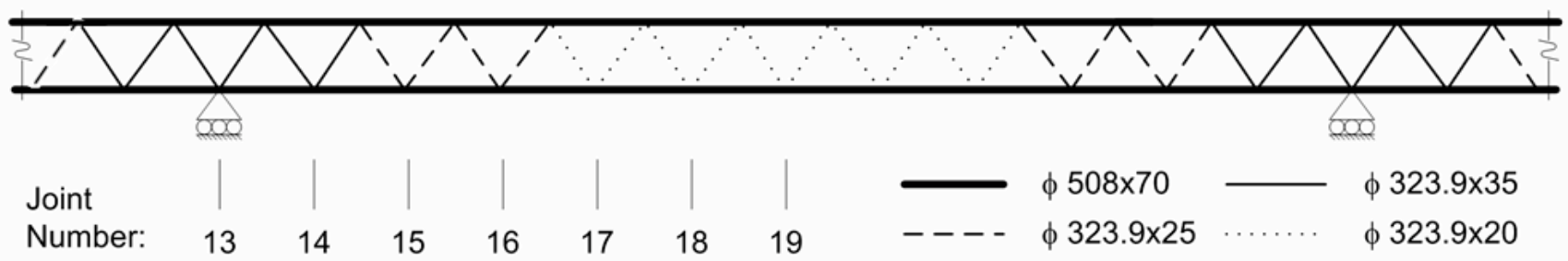

Figure 2. Example tubular bridge structure.

uniformly under-designed for fatigue, regardless of the value for $\gamma_{M f}$ assumed. Considering phase effects,

To determine the nominal member stresses, a simplified structural analysis was carried out wherein no interaction or load sharing between the two trusses was considered (i.e. each truss was assumed to support half of the dead load and half of the traffic load). The slab was assumed to be 300 $\mathrm{mm}$ thick over each truss, thinning to $250 \mathrm{~mm}$ at the edges and centre of the deck. Reinforcement ratios of $1 \%$ and $1.5 \%$ were assumed at the mid-span and over the support respectively. A modulus of elasticity ratio $\left(E_{\text {steel }} / E_{\text {concrete }}\right)$ of 10 was assumed. The slab was assumed to be cracked over the supports, meaning that only the reinforcement was assumed to contribute to the top chord stiffness in these areas.

At each hot-spot location, the equivalent design hot-spot stress range, $\Delta \sigma_{E 2}$, was calculated by taking the nominal member stress ranges for each of the five load cases in Figure 3, multiplying them by the appropriate $S C F \mathrm{~s}$, and summing the results to get the total hot-spot stress range, $\Delta \sigma\left(Q_{f a t}\right)$, as recommended in (Zhao et al. 2000). In employing this approach, phase effects were conservatively ignored, i.e. it was effectively assumed that the stress peaks for each load case occur at the same truck position.

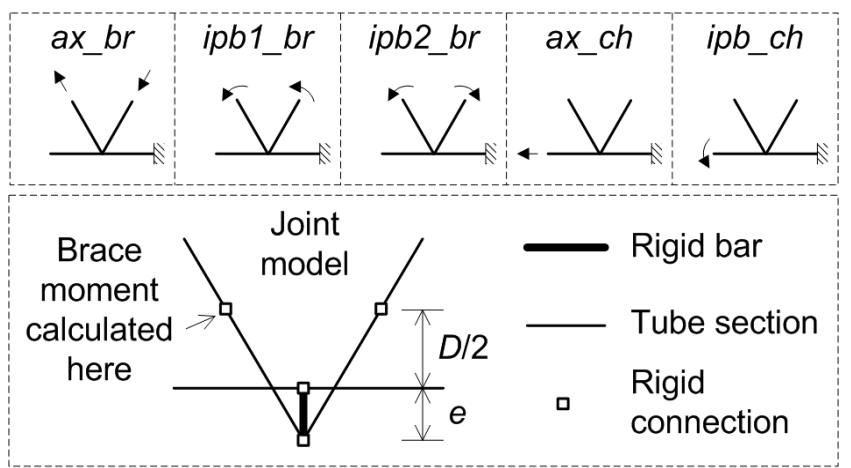

Figure 3. Joint model and nominal member load cases.

Using the verification approach described above, the member sizes in Figure 2 were selected, resulting in a bridge truss with joints that were more-or-less i.e. by first calculating the hot-spot stress for each truck position using Equation 4 and then determining the hot-spot stress range using the resulting hotspot stress influence line, was seen to improve the situation somewhat, as seen in Figure 4.

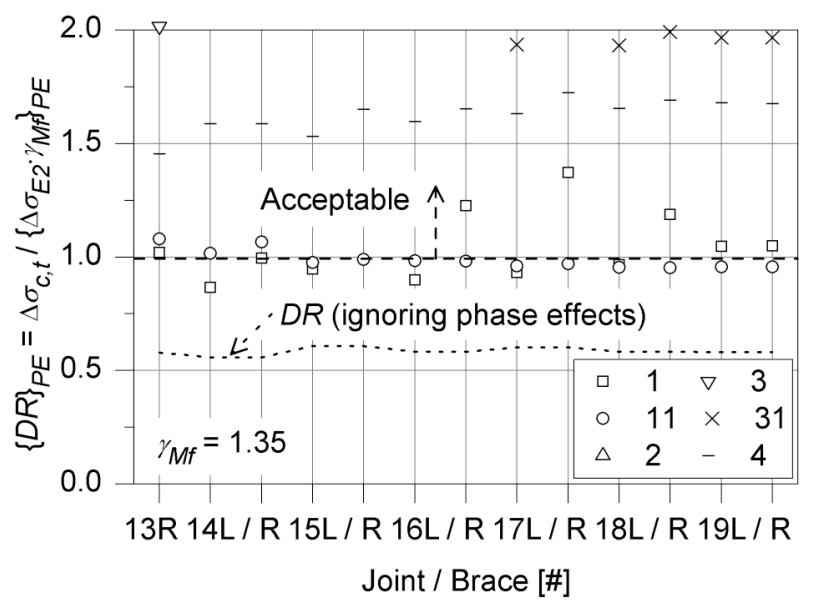

Figure 4. Design ratios, $\{D R\}_{P E}$, considering phase effects.

In this figure the results are presented of the deterministic verification considering phase effects (assuming $\gamma_{M f}=1.35$ ) for each hot-spot on each brace member of each joint along the bottom chord of the interior span (numbered as in Fig. 2). An envelope of the design ratio, $D R$, ignoring phase effects for the worst hot-spot on each brace member is included for comparison purposes.

From this figure, it can be deduced that the example structure can be made to pass the deterministic verification if phase effects are considered and a lower value for $\gamma_{M f}$ is permitted, such as 1.0. If use of the higher $\gamma_{M f}$ value is deemed necessary, then the example structure almost passes the verification if phase effects are considered, but fails by a considerable margin if these effects are ignored.

Also important to note in this figure is that the performance of each of the joints appears to be largely determined by Sites $1 \mathrm{~L}, 11 \mathrm{~L}, 1 \mathrm{R}$, and 11R. 


\section{PROBABILISTIC ANALYSIS}

Following the code-based fatigue design of the example bridge structure, a probabilistic analysis was conducted of each hot-spot (untreated) on each of the seven joints numbered in Figure 2.

To perform these analyses, the single site probabilistic model described in Appendix I was employed. As discussed in (Walbridge 2005), the required hot-spot stresses were determined in the same way as in the code-based verification considering phase effects. The required weld geometry parameters were determined at each hot-spot using parametric equations based on the AWS Code (2000).

In order to simulate realistic loading conditions, a traffic model was needed. The model eventually adopted consists of three truck types, with weight distributions as shown in Figure 5. The contribution of each truck type to the total traffic volume is also indicated in this figure. This model is based on weigh scale measurements taken on the main highway between Bern and Zurich, Switzerland (Kunz \& Hirt 1991), modified to consider the new 40 tonne Swiss legal truck weight limit as discussed in (Walbridge 2005). In using this model, the truck weights were multiplied by a dynamic factor of 1.3 .

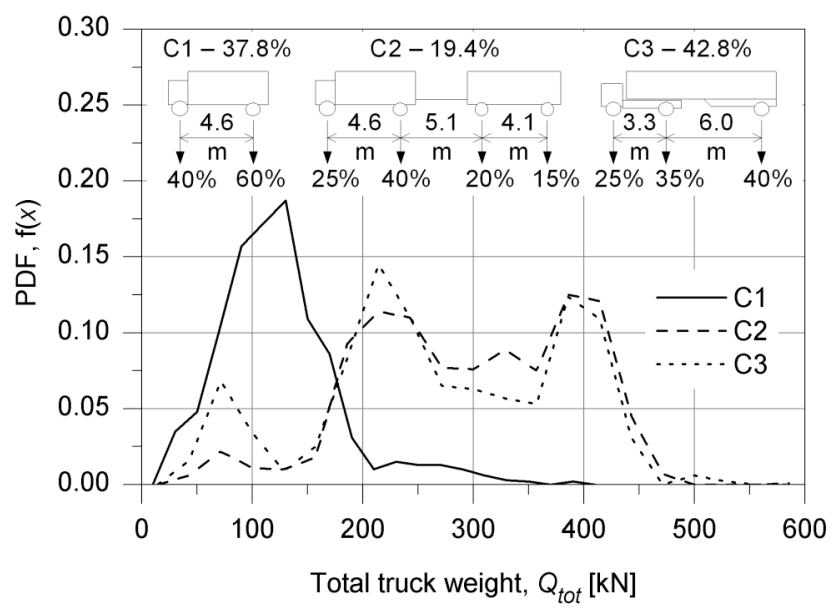

Figure 5. Traffic model used in probabilistic analysis.

The assumed values for the statistical variables required to apply the single site probabilistic model are summarized in Table 1. In this table the ' $V A R$ ' variables are typically factors by which the input parameters described by the subscripts are multiplied. The applied stresses due to the traffic and dead loads, for example, are multiplied by the variables: $V A R_{\text {traffic }}$ and $V A R_{\text {dead }}$. Regarding these variables, it should be pointed out that the mean values for Variables 2,12 , and 13 were determined by calibration using the test results from (Schumacher 2003). With these values, the model was seen to closely predict the mean and scatter of these test results.

To solve the resulting probabilistic fracture mechanics problem, a FORTRAN 90 subroutine employing the Monte Carlo simulation (MCS) solution method with a crude importance sampling scheme was used to determine the probabilities of failure, $p_{f}$, for the various hot-spots corresponding with the passage of a given number of trucks, $T r$.

With the resulting $p_{f}$ vs. $T r$ data, a reliability envelope was calculated for the interior span of one truss on the example bridge structure (see Fig. 6). This envelope takes into account the uncertainty in the true level of correlation between the probabilities of failure of the various hot-spots, as discussed in Appendix I. To produce this envelope, several approaches were considered to account for the top chord joints in the example structure. For illustrative purposes, the fatigue lives of the hot-spots in these joints are assumed herein to be sufficiently high that the fatigue reliability of the entire truss can be taken as roughly equal to that of the bottom chord.

Table 1. Statistical variables.

\begin{tabular}{llllll}
\hline Variable & $i$ & $\mu_{x}$ & $\sigma_{x}$ & Dist. & Units \\
\hline$a_{0}$ & 1 & 0.2 & 0.045 & $\mathrm{LN}$ & $\mathrm{mm}$ \\
$(a / c)_{0}$ & 2 & 0.5 & 0.16 & $\mathrm{LN}$ & - \\
$V A R_{\text {traffic }}$ & 3 & 1.0 & 0.15 & $\mathrm{~N}$ & - \\
$V A R_{\text {dead }}$ & 4 & 1.0 & 0.10 & $\mathrm{~N}$ & - \\
$V A R_{D O B}$ & 5 & 1.0 & 0.08 & $\mathrm{~N}$ & - \\
$V A R_{S C F}$ & 6 & 1.0 & 0.04 & $\mathrm{LN}$ & - \\
$V A R_{M k}$ & 7 & 1.0 & 0.05 & $\mathrm{LN}$ & - \\
$V A R_{L w}$ & 8 & 1.0 & 0.10 & $\mathrm{~N}$ & - \\
$V A R_{\theta w}$ & 9 & 1.0 & 0.10 & $\mathrm{~N}$ & - \\
$V A R_{\text {weld }}$ & 10 & 1.0 & 0.25 & $\mathrm{~N}$ & - \\
$V A R_{p w t}$ & 11 & 0.5 & 0.10 & $\mathrm{~N}$ & - \\
$\mathrm{LN}_{(C)}$ & 12 & -28.80 & 0.55 & $\mathrm{~N}$ & $\mathrm{LN}((\mathrm{mm} / \mathrm{cycle})$ \\
$\Delta K_{\text {th }}$ & 13 & 100.0 & 15.0 & $\mathrm{LN}$ & $\mathrm{MPa} \sqrt{ } \mathrm{mm}$ \\
$a_{c}$ & - & $0.5 \cdot T$ & - & det. & $\mathrm{mm}$ \\
$f_{y}$ & - & 355 & - & det. & $\mathrm{MPa}$ \\
$m$ & - & 3.0 & - & det. & - \\
\hline
\end{tabular}

Following the analysis of the untreated structure, a second series of calculations was performed with each potential crack site treated by needle peening. With the results of these calculations, a second reliability envelope was produced for the treated structure (see also Fig. 6). Comparing the envelopes for the untreated and treated structures, the potential benefit of the treatment could be determined.

Looking at Figure 6, it can be seen that the benefit of post-weld treatment was significant for the studied example bridge structure. In order to quantify this benefit, the fatigue lives of the untreated and treated bridges can be compared at a given target reliability index, $\beta_{\text {target }}$. In (Walbridge 2005), for example, comparisons are made at $\beta_{\text {target }}=3.74$. This is the target index suggested in the Eurocode EN 1990 (2002) for a structure with a design life of 70 years, a low level of redundancy, and limited possibility for inspection / repair. If this target index is used to compare the envelopes in Figure 6 , the benefit of treatment can be seen to be a 98 to $267 \%$ fatigue life 
improvement, depending on whether the results obtained with the upper or the lower bound series system models are used for the comparison.

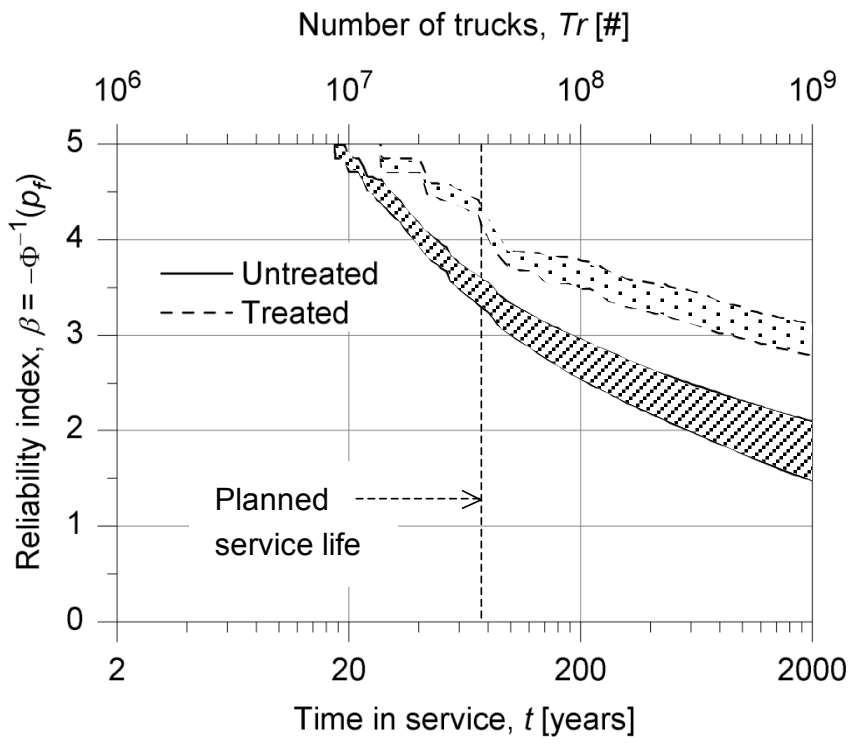

Figure 6. $\beta$ vs. Tr envelopes for example bridge structure.

Using the analytical approach described above a number of studies where carried out to determine the effects of variations in a number of the treatment parameters, as discussed in (Walbridge 2005). One useful result of these studies was the finding that the same treatment benefit could be achieved with a partial treatment strategy (TS4 in this reference), wherein only Sites 1 and 11 on the left and right braces are treated, as illustrated in Figure 7.

$\underline{\text { TS3 }}$ - full treatment

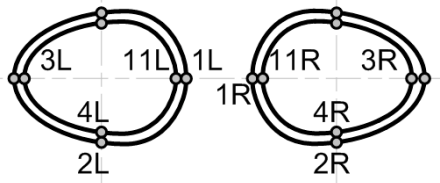

$\underline{\text { TS4 }}$ - Sites 1L, 11L $1 \mathrm{R}$, and $11 \mathrm{R}$ treated

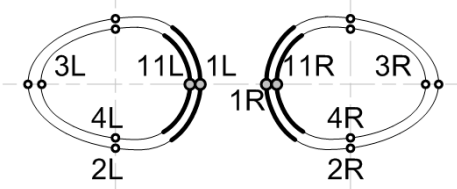

Figure 7. Full (TS3) and partial (TS4) treatment strategies.

This finding can be explained by examining the deterministic design ratios in Figure 4. Apparently post-weld treatment by needle peening is sufficient to increase the fatigue lives of Sites 1 and 11 to a certain extent, but this improvement is not so great as to make these sites less critical than the next most critical untreated site with treatment strategy TS4.

Another potentially useful result of the probabilistic calculations is that the single site results for the untreated bridge can be used to perform a verification of the deterministic, code-based design. The results of such a verification are summarized in Figure 8. In this figure, the design ratio considering phase effects, $\{D R\}_{P E}$, and assuming a low level of redun- dancy and limited possibility for inspection / repair $\left(\gamma_{M f}=1.35\right)$ is plotted verses the calculated fatigue life corresponding with $\beta_{\text {target }}=3.74$.

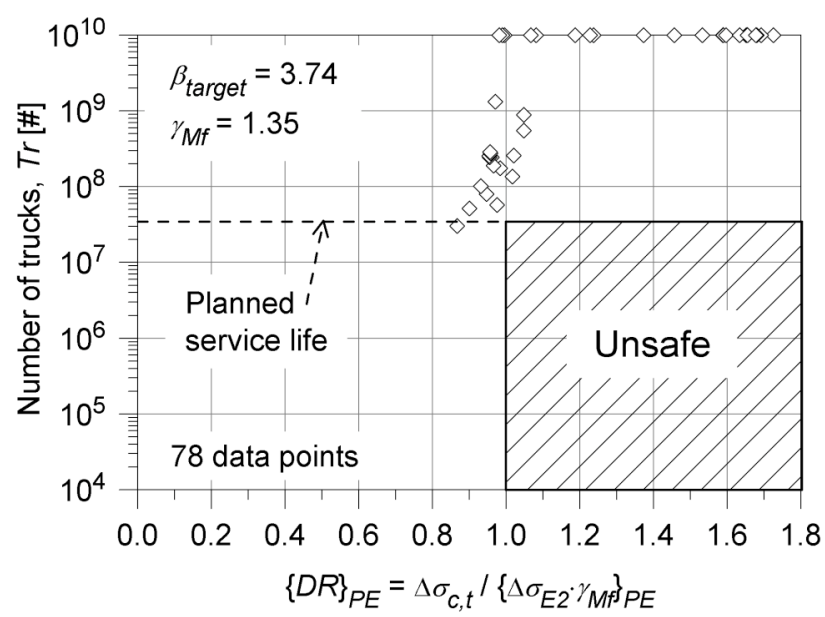

Figure 8. Verification of code-based design.

Looking at this figure, it can be concluded that according to the probabilistic model, the code-based design gives safe results for single potential crack sites, even when phase effects are considered. In other words, there is no case where the code-based design deems a potential crack site to be adequate, while the probabilistic model suggests it is not.

A number of the sites in Figure 8 are seen to perform much better than might be expected simply by looking at the deterministic design ratio. The main reason for this is that the code-based verification does not consider the beneficial effects of compressive stresses at the various potential crack site imposed by the dead loads. These stresses have a positive effect on the fatigue lives of these sites similar to that induced by the post-weld treatment.

As discussed in (Walbridge 2005), this verification is conservative in that the failure criterion for the probabilistic calculation is crack growth to a critical depth of $a_{c}=0.5 \cdot T$ (or $t$ ). In fact, tubular structures are known to possess a certain reserve capacity beyond crack growth to this depth. According to van Wingerde et al. (1997), for example, the time to total joint failure is on average 1.49 times as long as the time to through thickness cracking. In addition, a constant, but conservative $D O B$ has been assumed for all of the load cases in Figure 3.

One potentially unconservative assumption made by the probabilistic model is that the crack tip loading mode is essentially the same for all of the load cases in Figure 3 (i.e. primarily opening or Mode I loading). It is thought that the error due to this assumption should be small, although further study of the effect of the true crack tip loading mode may be of value if phase effects are to be routinely considered in the code-based design. The results of this further study are not, however, expected to have serious implications on the calculated benefit of postweld treatment as seen, for example, in Figure 6. 


\section{WELD ROOT VERIFICATION}

One potential concern with the use of post-weld treatment methods is the increased possibility, with treatment, that the eventual failure of the joint will result from fatigue cracking at the weld root. Cracking at this location is generally considered to be much less desirable, as there is no possibility in this case for early detection by visual inspection.

One possible fatigue assessment approach for the weld root, inspired by the hot-spot stress approach commonly used for the weld toe, is discussed in (Health and Safety Executive 1999). This reference provides a set of parametric equations for a factor, $R_{S C F}$, which relates the maximum $S C F$ at the weld root to that at the weld toe, i.e.:

$R_{S C F}=\frac{S C F_{\text {root, } \max }}{S C F_{\text {toe, } \max }}$

These equations require as input the following parameters: $\beta, \gamma, \tau, \zeta(=g / D)$, and $\theta_{b r}$. Equations are provided for two of the brace load cases $(a x b r$ and $i p b 2$ br) in Figure 3. As is often the case with research conducted primarily for the offshore industry, the validity range for the $\gamma$ parameter is much higher $(12 \leq \gamma \leq 30)$ than the range for this parameter common to tubular bridge structures. If this range is ignored, however, these equations can be applied to the example bridge to determine the possible implications of weld root cracking. The results of such an application are summarized in Figure 9.

To generate the design ratios for the weld root in this figure, it was assumed that $R_{S C F}$ was the same for both brace bending load cases in Figure 3. Furthermore, it was assumed that the chord load cases would not affect $R_{S C F}$. On this basis, a hot-spot stress range for the weld root was calculated. Phase effects were ignored in this calculation because it was necessary to separate the effects of the various load cases to apply these equations. The applied stress range was then compared to a fatigue strength at $2 \cdot 10^{6}$ applied stress cycles, $\Delta \sigma_{c, t}$, of $90.6 \mathrm{MPa}$ for the weld root, as proposed in (Health and Safety Executive 1999), corrected for size using Equation 3, assuming (conservatively) that the weld root crack will occur in the thicker chord wall.

Looking at Figure 9, it can be concluded that cracks will most likely always initiate from the weld toe in the untreated bridge. In this figure, a theoretical design ratio is presented for the bridge treated using strategy TS4. As the deterministic design ratios for the treated sites could not be calculated, these sites were simply not considered in the determination of the design ratios for the joints, effectively modelling the case of perfect treatment. Comparing the data for the treated weld toe and the untreated root, it can be seen that the post-weld treatment should not result in a shift in the critical crack site from the weld toe to the weld root if this partial treatment strategy is employed.

To make sure of this, it is recommended that the weld root be designed with a fatigue life 100 to $200 \%$ greater than that of the weld toe (Health and Safety Executive 1999). This can be equated to ensuring that the design ratio of the root is 1.25 to 1.44 that of the toe if an S-N curve slope of $m=3.0$ is assumed. A fourth curve in Figure 9 shows the maximum allowable design ratio for the weld toe using the more severe of these two limits. As can be seen in this figure, the benefit of treatment using strategy TS4 can still be deemed permissible on this basis.

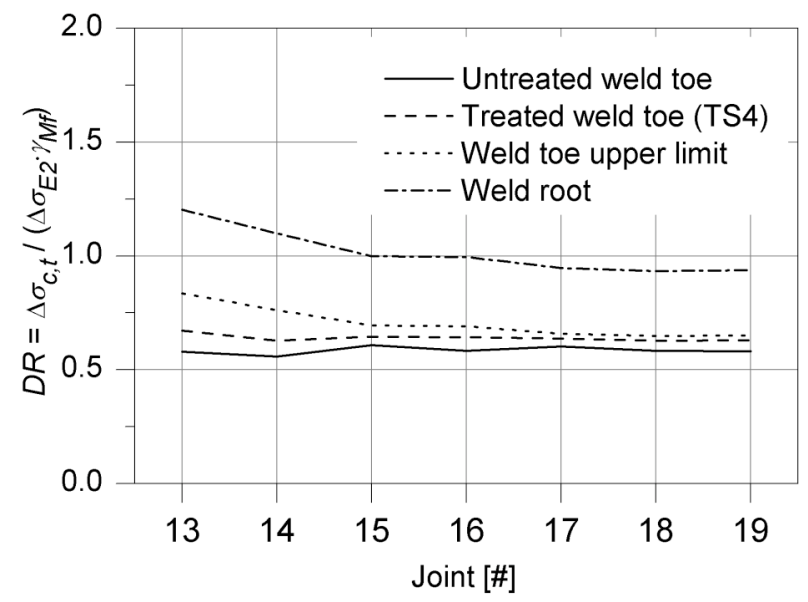

Figure 9. Deterministic weld root verification.

\section{CONCLUSIONS}

The analyses presented herein demonstrate the potential of post-weld treatment methods such as needle peening for increasing the fatigue lives of tubular bridge structures. Herein, it is also shown that the same treatment benefit can be achieved for tubular bridges similar to the studied example bridge structure with a partial treatment strategy that would likely be much less costly than full treatment.

Finally, a deterministic verification of the weld root is presented. Although this verification shows that the adopted partial treatment strategy will not cause the critical crack location to shift to the weld root, further work is clearly needed to develop parametric equations for the $R_{S C F}$ factor that are adapted to tubular joints with geometries typical of bridge structures. In addition, there may be value in modifying the weld root verification procedure so that phase effects can be more easily considered.

\section{ACKNOWLEDGEMENTS}

The work presented herein was supported by the Swiss Federal Highway Administration (OFROU Project No. AGB2002/011) and the Swiss National Research Foundation (SNF Grant 200020-101521). 


\section{APPENDIX I - PROBABILISTIC MODELS}

\subsection{Single site model}

The limit state function, $\mathrm{G}(\mathbf{z})$, for the probabilistic single site model employed herein is founded on the Paris-Erdogan crack growth law, modified to consider crack closure effects and a threshold stress intensity factor (SIF) range, $\Delta K_{t h}$, and integrated over a crack depth range, $a_{0}$ to $a_{c}$. Specifically:

$$
G(\mathbf{z})=N_{c}-N=\int_{a_{0}}^{a_{c}} \frac{d a}{C \cdot\left(\Delta K_{e f f}{ }^{m}-\Delta K_{t h}{ }^{m}\right)}-N
$$

where:

$$
\begin{aligned}
\Delta K_{\text {eff }} & =\operatorname{MAX}\left(K_{a p p, \max }-K_{o p}, 0\right) \\
& -\operatorname{MAX}\left(K_{a p p, \min }-K_{o p}, 0\right)
\end{aligned}
$$

where $K_{a p p, \max }$ and $K_{a p p, \min }=$ maximum and minimum SIFs due to the applied load; and $K_{o p}=$ applied SIF at which crack tip opens upon loading.

Herein, $K_{o p}$ is calculated as follows:

$$
K_{o p}=-\left(K_{r e s}+K_{p l}\right)
$$

where $K_{\text {res }}=$ SIF due to residual stress distribution along anticipated crack path; and $K_{p l}=$ crack closure SIF. Herein, $K_{p l}$ is calculated using the following empirical expression from (Bremen 1989):

$$
\begin{aligned}
K_{p l}= & -\operatorname{MIN}\left(0.2 /\left(1-R_{e f f}\right), 0.28\right) \\
& \cdot\left(K_{a p p, \max }+K_{\text {res }}\right)
\end{aligned}
$$

where $R_{\text {eff }}=$ effective stress ratio. Specifically:

$R_{e f f}=\frac{K_{a p p, \min }+K_{r e s}}{K_{a p p, \max }+K_{r e s}}$

$K_{\text {res }}$ is calculated at each crack depth increment using the approach proposed by Albrecht \& Yamada (1977). The assumed stress distributions needed for this approach are based on those proposed by Stacey et al. (2000) and Bremen (1989) for the welding and post-weld treatment (PWT) residual stresses.

The assumed residual stress distribution due to the welding process is as follows:

$$
o_{\text {weld }}(b)=f_{y} \cdot\left(\begin{array}{l}
0.62+2.33 \cdot(b / T) \\
-24.13 \cdot(b / T)^{2} \\
+42.49 \cdot(b / T)^{3} \\
-21.09 \cdot(b / T)^{4}
\end{array}\right) \cdot V A R_{\text {weld }}
$$

where $b=$ depth below surface; and $T=$ wall thickness of cracked member. In this expression, $V A R_{\text {weld }}$ is a statistical variable introduced to consider the uncertainties associated with this stress distribution.
The assumed PWT residual stress distribution is:

$$
\begin{aligned}
\dot{o}_{p w t}(b) & =-f_{y} \cdot\left(V A R_{p w t}\right) \text { if } b \leq 0.1 \cdot d_{p} \\
& =f_{y} \cdot\left(\begin{array}{l}
\left(b / d_{p}\right) \cdot(5 / 6)- \\
\left(1 / 12+V A R_{p w t}\right)
\end{array}\right) \text { if } b>0.1 \cdot d_{p}
\end{aligned}
$$

where $d_{p}=$ imprint diameter of peening tool $(1.5 \mathrm{~mm}$ for needle peening). To determine the combined (welding plus treatment) stress distribution, $\sigma_{\text {res }}(b)$, the parameter $P W T$ is introduced, such that:

$$
\begin{aligned}
o_{\text {res }}(b) & =\operatorname{MIN}\left(o_{p w t}(b), o_{\text {weld }}(b)\right) \text { if } P W T=1 \\
& =o_{\text {weld }}(b) \text { if } P W T=0
\end{aligned}
$$

To solve Equation A2, $K_{\text {app }}$ is determined using the following expression from (Bowness \& Lee 1999):

$$
K_{a p p}=\left(\begin{array}{l}
M k_{m} \cdot Y_{m} \cdot(1-D O B) \\
+M k_{b} \cdot Y_{b} \cdot D O B
\end{array}\right) \cdot \sigma_{h s, a p p} \cdot \sqrt{\pi \cdot a}
$$

where $\sigma_{h s, a p p}=$ applied hot-spot stress; $M k_{m}, M k_{b}, Y_{m}$, and $Y_{b}=$ magnification and correction factors for the bending $\left(\sigma_{b}\right)$ and membrane $\left(\sigma_{m}\right)$ stress cases; and $D O B=$ degree of bending $\left(=\sigma_{b} /\left(\sigma_{b}+\sigma_{m}\right)\right)$.

In using this approach, it is essentially assumed that a weld toe crack anywhere on a tubular joint behaves in the same way as a similar crack in a T-butt joint such as the one in Figure A1.

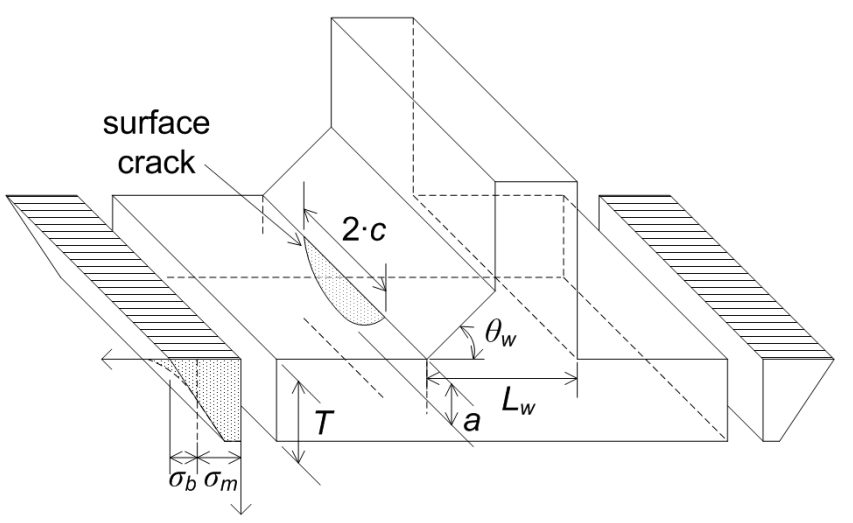

Figure A1. Crack at weld toe of T-butt joint.

Herein, $M k_{m}$ and $M k_{b}$ are solved using parametric equations from (Bowness \& Lee 1999). These equations require as input the following parameters: $a / T$, $a / c, L_{w} / T$, and $\theta_{w} . Y_{m}$ and $Y_{b}$ are solved using parametric equations from (Newman and Raju 1981). These equations require as input the following parameters: $a / T$ and $a / c$. The $D O B$ is solved using parametric equations from (Connolly et al. 1990), making assumptions for the missing load cases, and ignoring the limits on $\gamma$. These equations require as input the hot-spot location along with the following parameters: $\alpha\left(=2 \cdot L_{c h} / D\right), \beta, \gamma, \tau$, and $\theta_{b r}$.

A one-dimensional crack propagation model is employed herein with the aspect ratio, $a / c$, varied according to a predefined crack shape evolution function wherein the initial aspect ratio, $(a / c)_{0}$, may 
vary, but this ratio then evolves smoothly, converging on a fixed value of 0.2 at $b / T=0.25$.

\subsection{Multiple site model}

In order to determine the probabilities of failure of structures comprised of tubular K-joints with multiple potential crack sites, lower and upper bound reliability models for series systems are employed herein. Specifically, it is first assumed that each Kjoint in the structure can be modelled as a series system with 16 constituent elements, corresponding with each of the hot-spots identified in Figure 1 (Note: Sites 2L, 4L, 2R, and 4R each occur twice).

The lower bound model assumes that the probabilities of failure of the individual hot-spots are fully independent. On this basis the probability of failure of the joint can be written as follows:

$$
p_{f, j o i n t}=1-\left(1-p_{f, I L}\right) \cdot\left(1-p_{f, I L L}\right) \cdot \ldots \cdot\left(1-p_{f, 4 R}\right)
$$

where $p_{\text {fjoint }}=$ probability of joint failure; $p_{f, 1 L}=$ probability of failure of Site $1 \mathrm{~L}$, etc. To determine the probabilities of failure of tubular structures with multiple joints, a similar approach is employed.

The upper bound reliability model assumes full correlation of the probabilities of failure at each potential crack site, and takes the following form:

$$
p_{f, j o i n t}=\operatorname{MAX}\left(p_{f, I L}, p_{f, I L}, \ldots, p_{f, 4 R}\right)
$$

\subsection{Additional considerations for analysis under realistic loading conditions}

As the probabilistic single site model is presented in Section 8.1, it can only be used for analysis under constant amplitude (CA) loading conditions.

In order to analyze structures under realistic, variable amplitude loading conditions, modifications to the adopted fracture mechanics model are therefore required. A number of candidate models for predicting crack growth rates under variable amplitude (VA) loading conditions are summarized in (Stephens et al. 2001). Among the simplest of these methods are the Equivalent constant amplitude stress range and Equivalent block loading approaches. Dubois (1994) and Manteghi \& Maddox (2004) both found that fatigue life predictions made using the first approach are often highly unconservative for post-weld treated details. For this reason, the second approach is adopted herein.

According to this approach, the VA stress spectrum is divided into a number of CA stress range blocks. At each crack depth, $a$, the crack closure SIF due to the maximum stress for each block is calculated. The rate of crack growth is then determined herein by calculating the damage due to each CA stress range block, assuming a crack closure SIF for all blocks equal to the largest crack closure SIF caused by any one block at that crack depth.

\section{REFERENCES}

Albrecht, P. \& Yamada, K. 1977. Rapid Calculation of Stress Intensity Factors. Journal of Structural Engineering 103(ST2):377-389.

American Welding Society 2000. ANSI/AWS D1.1-2000 Structural Welding Code-Steel. U.S.A.

Bowness, D. \& Lee, M.M.K. 1999. Weld Toe Magnification Factors for Semi-Elliptical Cracks in T-Butt Joints. Offshore Technology Report - OTO 199 014, Health and Safety Executive (HSE), U.K.

Bremen, U. 1989. Amélioration du comportement à la fatigue d'assemblages soudés : étude et modélisation de l'effet de contraintes résiduelles. EPFL Thesis No. 787, Lausanne.

Connolly, M.P., Hellier, A.K., Dover, W.D., \& Sutomo, J. 1990. A parametric study of the ratio of bending to membrane stress in tubular Y- and T-joints. International Journal of Fatigue 1:3-11.

Dubois, V. 1994. Fatigue de détails soudés traités sous sollicitations d'amplitude variable. EPFL Thesis No. 1260, Lausanne.

European Committee for Standardization 2002. EN1990 - Basis of structural design. Brussels.

Health and Safety Executive 1999. Fatigue Life Implications for Design and Inspection for Single Sided Welds at Tubular Joints. HSE Report No. OTO 99/022, U.K.

Kunz, P. \& Hirt M.A. 1991. Grundlagen und Annahmen für den Nachweis der Ermüdungsfestigkeit in den Tragwerksnormen des SIA. Doc. D 076, Swiss Society of Engineers and Architects (SIA), Zurich.

Manteghi, S. \& Maddox, S.J. 2004. Methods for fatigue life improvement of welded joints in medium and high strength steels. Doc. XIII-2006-04, International Institute of Welding (IIW).

Newman, J.C. \& Raju, I.S. 1981. An Empirical Stress-Intensity Factor Equation for the Surface Crack. Engineering Fracture Mechanics 15(1-2):185-192.

Romeijn, A., Karamanos, S.A., \& Wardenier, J. 1997. Effects of joint flexibility on the fatigue design of welded tubular lattice structures. 7th International Offshore and Polar Engineering Conference.

Schumacher, A. 2003. Fatigue behaviour of welded circular hollow section joints in bridges. EPFL Thesis No. 2727, Lausanne (http://icom.epfl.ch).

Stacey, A., Barthelemy, J.-Y., Leggatt, R.H., \& Ainsworth, R.A. 2000. Incorporation of Residual Stresses into the SINTAP Defect Assessment Procedure. Engineering Fracture Mechanics 67:573-611.

Stephens, R.I., Fatemi, A., Stephens, R.R., \& Fuchs, H.O. 2001. Metal Fatigue in Engineering (2nd Ed.). John Wiley $\&$ Sons.

Swiss Society of Engineers and Architects (SIA) 2003. SIA 260/261/263:2003 (Basis for structural design/Actions on structures/Steel structures). Zurich.

van Wingerde, A.M., van Delft, D.R.V., Wardenier, J., \& Packer, J.A. 1997. Scale Effects on the Fatigue Behaviour of Tubular Structures. WRC Proceedings, International Institute of Welding 123-135.

Walbridge, S. 2005. A probabilistic study of fatigue in postweld treated tubular bridge structures. EPFL Thesis No. 3330, Lausanne (http://icom.epfl.ch).

Walbridge, S., Nussbaumer, A., \& Hirt, M.A. 2003. Fatigue Behaviour of Improved Tubular Bridge Joints. Proceedings of the $10^{\text {th }}$ International Symposium on Tubular Structures, Madrid.

Zhao, X. L., Herion, S., Packer, J. A., et al. 2001. Guide No. 8 - Design guide for circular and rectangular hollow section joints under fatigue loading. Cologne: CIDECT. 